\title{
Oblique Incidence lonospheric Sounding: A Bibliometric Research
}

\section{J. T. D. Sartori}

\begin{abstract}
The ionosphere is one of the layers of Earth's atmosphere and is of special interest for telecommunications that pass through it or are reflected by it. Among other factors, it is specially affected by the Sun, causing variations in the layer height, ionization, electron density, and total electron content. As a result of these variations, the critical frequency also varies as well as propagation properties. The precise positioning of global navigation satellite systems is also affected by ionospheric properties. For those reasons, there are numerous high-frequency radars (ionosondes) worldwide dedicated to making the vertical sounding of the ionosphere (local measurements of its properties). Due to cost and complexity, there is a limited number of observatories, and a global ionospheric map requires modeling and inference for global coverage. Thus, oblique incidence sounding has the potential of contributing to further expand local readings without the need of establishing additional stations, being in theory an optimized way to improve existing ionospheric mapping and prediction. To advance toward that goal, a bibliometric research was conducted to retrieve, present, and analyze the metrics of existing publications on the topic. As a result, 862 unique papers were identified, ranging from 1936 to 2021, with evidence of increasing research interest in the past 15 years. In addition, a network analysis of coauthorship and co-occurrence is presented.
\end{abstract}

\section{Introduction}

The Earth's ionosphere is the charged part of the atmosphere that occurs between approximately 60 and $1000 \mathrm{~km}$. Variations in its properties affect most types of communications that travel within or across the atmosphere as they change propagation characteristics and usage, including radio and global navigation satellite systems.

Among other factors, the Sun is the main source of those fluctuations, especially through its flares and coronal mass ejections [3,4]. Thus, its study is a key factor to accessing and forecasting ionosphere $[5,6]$. In addition, it is well understood how temperature affects the ionosphere's thickness, density, and altitude [7-9].

That knowledge has grown in parallel and as a consequence of the evolution of electronics and communications, especially in the second half on the 20th century. And from that evolution, initiatives like the International Reference Ionosphere [10] have been

Manuscript received 17 November 2021.

Jeanfrank Teodoro Dantas Sartori is with UFPR (Federal University of Paraná), 632 Avenida Prefeito Lothário Meissner, Curitiba, Paraná, 80210-170, Brazil; e-mail: jeanfrank@ufpr.br. developed to produce models of the ionosphere based on different available sources, especially the large data sets obtained from ionosonde networks.

Although there has been intense research of the ionosphere and a wide development of applications, still not fully explored is the potential for the use of oblique sounding [11], which is why the present study aims to contribute to advances in that perspective by conducting a bibliometric research of scientific publications on the topic as one of the initial steps in that process.

\section{Methods}

Bibliometrics, from the Greek terms biblion ("book") and metrikos ("measurement"), is a research method first applied in 1917 [12] that evolved from an initial focus on evaluating a library collection to a more contemporary usage to describe the research corpus in an area or topic, helping to understand its evolution and current state. The focus of bibliometrics, especially in terms of occurrences, frequencies, and relationship networks, is on journals, authors, and key words, what has been classically described, respectively, as its three laws: Bradford's law, Lotka's law, and Zipf's law [13-16].

This bibliometric study aims to retrieve, present, and analyze currently available research on the topic of ionospheric oblique incidence sounding. The search of publications was conducted in September 2021 on the Web of Science and Scopus academic databases for being the two largest, most far reaching, and most widely used worldwide.

On both databases, we used the advanced query "(((oblique AND sounding) OR (passive AND sounding) OR (oblique AND incidence) OR (oblique AND ionogram) OR (oblique AND ionograms) OR (oblique AND ionosonde) OR (oblique AND ionosondes) OR (oblique AND digisonde) OR (oblique AND digisondes)) AND (ionosphere OR ionospheric OR ionogram OR ionograms))," considering only title, abstract, and authors' key words ("TS" criteria on Web of Science and "TITLE-ABS-KEY" on Scopus).

The search resulted in an initial corpus of 1284 publications retrieved (516 on Web of Science and 768 on Scopus). From that resulted a total of 862 unique documents, using JabRef 5.3 for consolidation; this consisted of 693 articles, 164 conferences/proceedings, and five books.

For producing the network view of coauthorship and of key word co-occurrences, VOSViewer software has been used, using references in the RIS format [17]. For graphs and tables of the frequency of publications per journal and per year, JabRef software was used to export the references from the RIS format to the CSV 


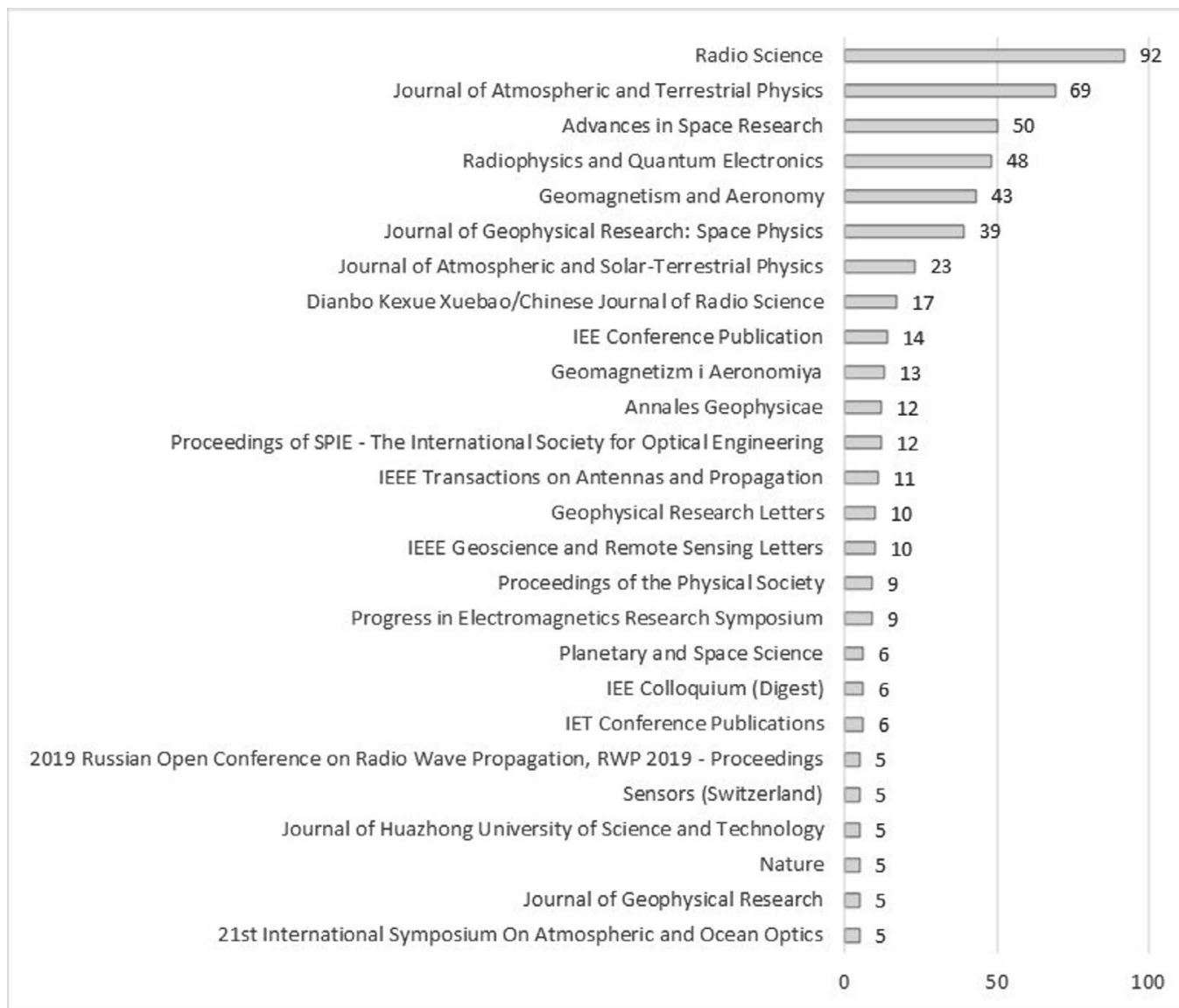

Figure 1. Number of publications per journal or event with five or more occurrences.

format, which was then imported into Microsoft Excel, where the information was summarized and the graphs and tables were generated.

\section{Results and Discussion}

A total of 257 different journals and conferences have been identified among the retrieved publications. In addition, it is possible to observe an increase in the number of publications in the past 15 years, with 2009 and 2019 being the two years with most publications.
In terms of journals, Figure 1 presents a graphical summary for those with the number of publications equal to or higher than 5 . Three journals presented more than 50 publications-Radio Science (92, 11\%), Journal of Atmospheric and Terrestrial Physics (69, 8\%), and Advances in Space Research (50, 6\%) - while 167 had a single occurrence each. In addition, 12 journals had between 10 and 49 publications each, and 73 had between two and nine publications each.

When investigating the numbers of publications per year, as presented in Figure 2, the initial work

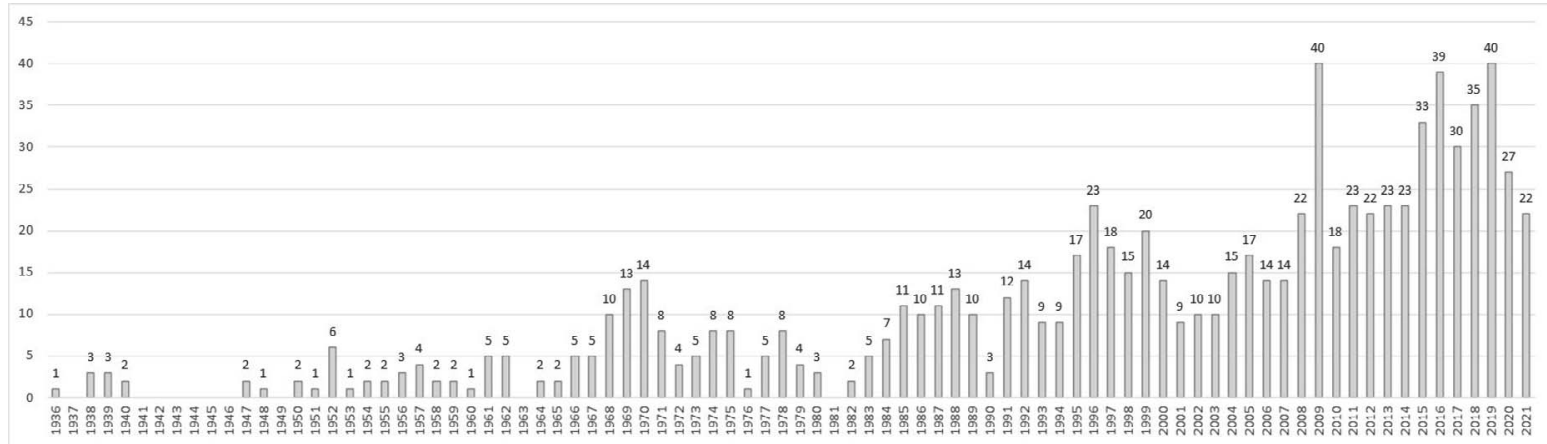

Figure 2. Publications per year. 
Table 1. Types of authorship of the publications

\begin{tabular}{lrccr}
\hline $\begin{array}{l}\text { Type of authorship and } \\
\text { number of authors }\end{array}$ & Publications & \multicolumn{3}{c}{$\%$ of the total } \\
\hline Shared authorship & 1 & 0.1 & 29.4 & 84.6 \\
31 & 1 & 0.1 & & \\
22 & 1 & 0.1 & & \\
19 & 3 & 0.3 & & \\
15 & 2 & 0.2 & & \\
14 & 3 & 0.3 & & \\
13 & 2 & 0.2 & & \\
12 & 6 & 0.7 & & \\
11 & 3 & 0.3 & & \\
10 & 17 & 2.0 & & \\
9 & 21 & 2.4 & & \\
8 & 36 & 4.2 & & \\
7 & 61 & 7.1 & & \\
6 & 96 & 11.1 & & \\
5 & 133 & 15.4 & 70.6 & \\
4 & 166 & 19.3 & & \\
3 & 177 & 20.5 & & \\
2 & & & & 15.4 \\
Single author & 133 & 15.4 & & \\
1 & 862 & 100 & 100 & 100 \\
Total & & &
\end{tabular}

emerged in the early 1930s, and since then a few gaps have occurred, those being years without papers on oblique incidence soundings: 1937, 1941-1946, 1949, 1963, and 1981. Since then, every single year had at least one publication.

The citation analysis (for Scopus available only from 1970 and for Web of Science from 1945) of those publications, being the number of times in each year that the select corpus has been cited, shows a growing trend, the vast majority being non-self-citations, also representing a persistent growth in the number of researchers. An h-index of 27 is observed in both Web of Science and Scopus. It is interesting to note an intense increase in the past 15 years in the citations of publications of journals indexed by both databases.

In terms of types of authorship, as shown in Table 1 , most $(84.6 \%)$ correspond to publications with two or more researchers, with 133 publications (15.4\%) being
Table 2. Authors with 15 or more publications on the topic

\begin{tabular}{lrlc}
\hline Author & Publications & \multicolumn{1}{c}{ Author } & Publications \\
\hline Zhao, Z. & 42 & Zhao, Z.-Y. & 19 \\
Chen, G. & 33 & Zhou, C. & 19 \\
Uryadov, V. P. & 32 & Yang, G. & 18 \\
Kurkin, V. I. & 29 & Vertogradov, G. G. & 18 \\
Ponomarchuk, S. N. & 22 & Grozov, V. P. & 17 \\
Blagoveshchensky, D. V. & 20 & Ivanov, V. A. & 16 \\
Krasheninnikov, I. V. & 19 & Chernogor, L. F. & 15
\end{tabular}

single authored; $29.4 \%$ of the papers present five or more authors, with the publications with the most authors (30) dating from 2005. Two authors is also the most common number of coauthors, corresponding to 20.5\% (177 publications).

Table 2 presents authors with most publications. It can be highlighted that $36.6 \%$ of authors from the retrieved papers have a single publication on the topic and that $15.0 \%$ have only two, meaning that a substantial part seems to not extend their research on this topic.

From the analysis of key word co-occurrences, it is possible to observe in Figure 4 that there are many different clusters associated. In addition, from the analysis of coauthorship, it is possible to observe in Figure 5 the main clusters of researchers at the center, formed mainly by the authors in Table 3 . In addition, it can be identified that some of these clusters do not interconnect. Also, there are some minor clusters and independent authors shown in gray.

\section{Final Considerations}

This work investigated publications on the topic of oblique incidence sounding of the ionosphere using the bibliometric method. Its main goal was to identify, present, and analyze the metrics of existing publications, resulting in 862 unique ones being retrieved, ranging between 1936 and 2021.

It has been identified that in recent years, a growth has occurred in the number of publications on oblique

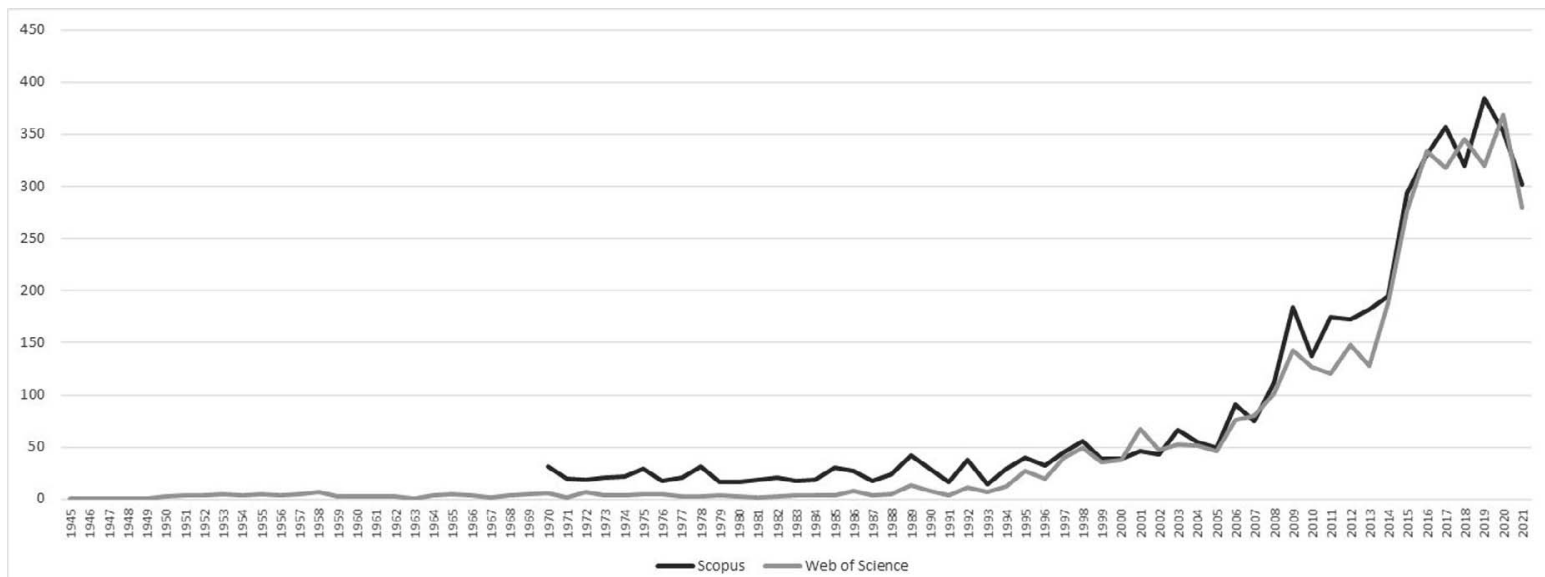

Figure 3. Yearly number of times the selected publications were cited. 


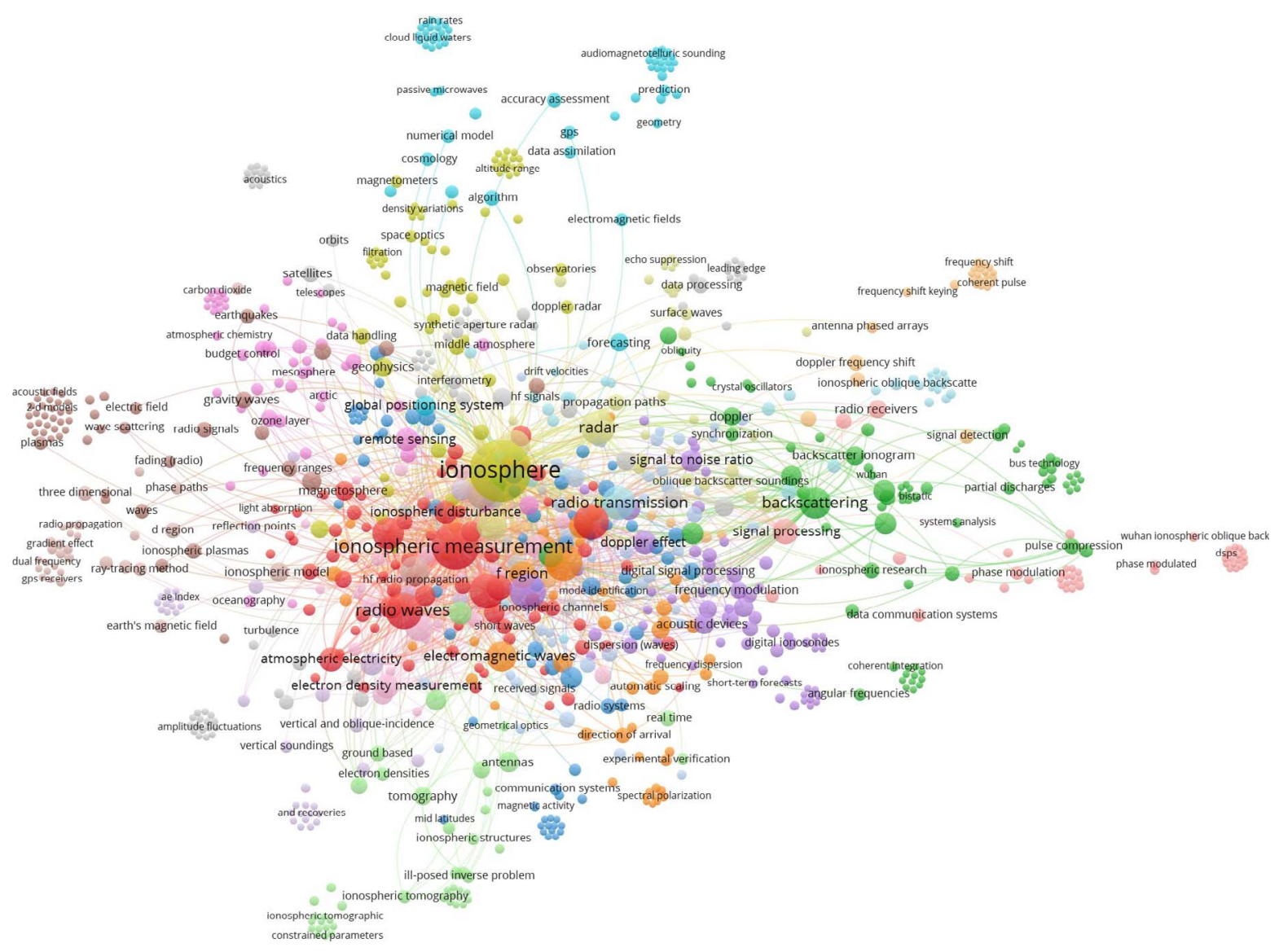

Figure 4. Network view of key word co-occurrences.
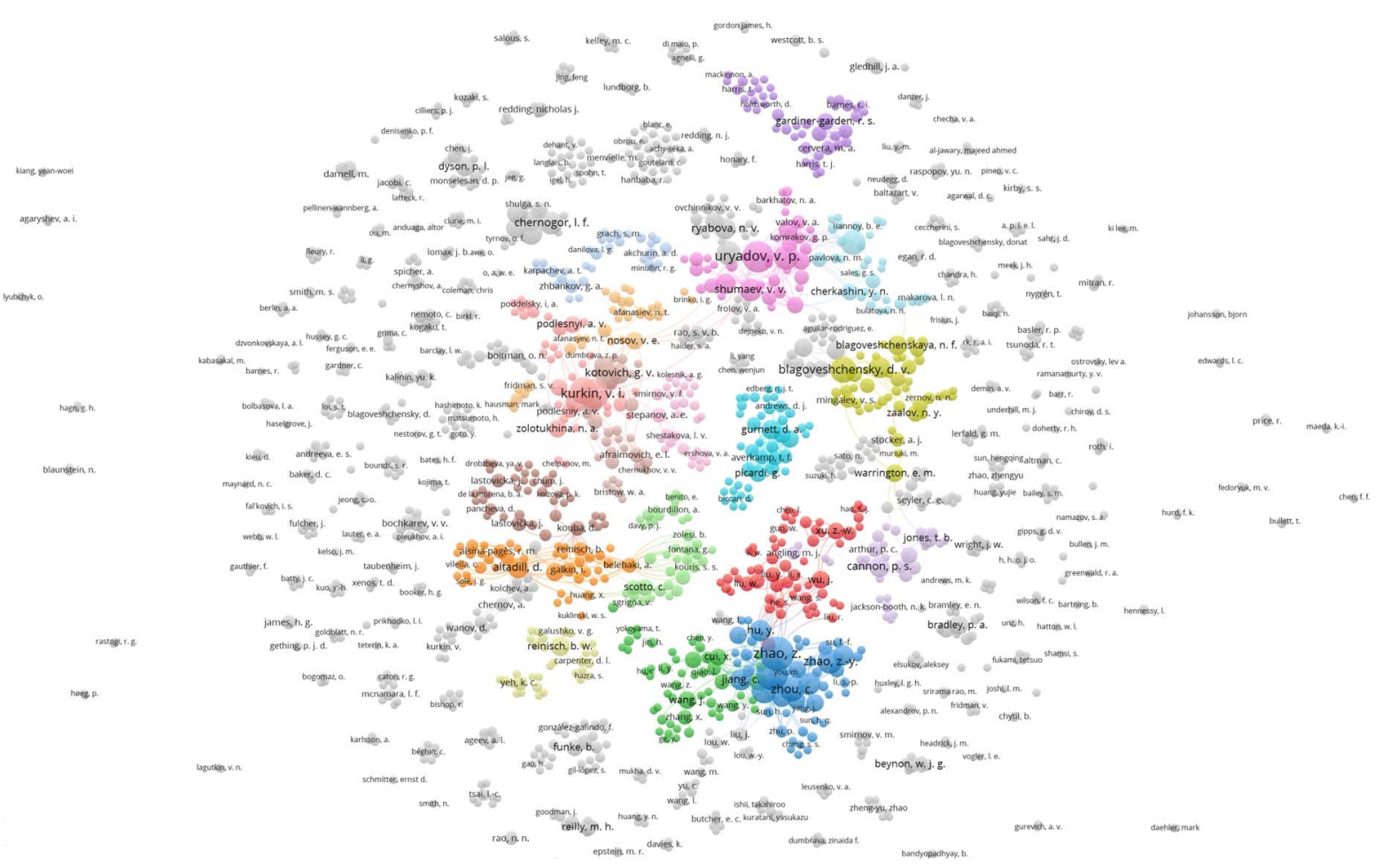

Figure 5. Network view of coauthorship. 
incidence sounding resulting from an increased research interest in the academic community. That fact reinforces the potential and viability of the use of that modality for upgrading ionospheric modeling, description, and prediction, as there is already a substantial developed background and is trending better in the research field.

On the other hand, that may also indicate that the topic is far from being consolidated, and many of its challenges are yet to be overcome, as highlighted in recent studies and experiments [18]. Further evidence of this consolidation need emerges from the fact that there are many coauthorship cluster groups that are not linked, thus possibly representing distinct research branches on the topic. There are also many other relevant authors who did not form clusters in the topic and are not connected to any cluster groups.

Thus, it is proposed that more scientific effort should be dedicated to investigating and solving those existing challenges, as the broad and systematic use of oblique incidence sounding has a high potential to contribute to further improving the capabilities of measuring, modeling, understanding, and predicting the ionosphere with a special focus on propagationrelated properties. In addition, it is proposed that future research conduct a literature review of the state of the art on the subject, dedicating special effort to unify what appears to be different branches of studies that might not be currently properly linked or consolidated.

\section{References}

1. J. M. Goodman (ed.), The Effect of the Ionosphere on Communication, Navigation, and Surveillance Systems, Washington, DC, Naval Research Laboratory, 1988.

2. D. Bilitza, D. Altadill, V. Truhlik, V. Shubin, I. Galkin, B. Reinisch, and X. Huang, "International Reference Ionosphere 2016: From Ionospheric Climate to Real-Time Weather Predictions," Space Weather, 15, 2, 2017, pp. 418-429.

3. N. Buzulukova (ed.), Extreme Events in Geospace: Origins, Predictability, and Consequences, Amsterdam, Elsevier, 2017.

4. M. Piersanti, T. Alberti, A. Bemporad, F. Berrilli, R. Bruno, et al., "Comprehensive Analysis of the Geoeffective Solar Event of 21 June 2015: Effects on the Magnetosphere, Plasmasphere, and Ionosphere Systems," Solar Physics, 292, 11, 2017, pp. 1-56.
5. F. Berrilli, M. Casolino, D. Del Moro, L. Di Fino, M. Larosa, et al., "The Relativistic Solar Particle Event of May 17th, 2012 Observed On Board the International Space Station," Journal of Space Weather and Space Climate, 4, 2014, p. A16.

6. L. Di Fino, V. Zaconte, M. Stangalini, R. Sparvoli, P. Picozza, et al., "Solar Particle Event Detected by ALTEA On Board the International Space Station-The March 7th, 2012 X5.4 Flare," Journal of Space Weather and Space Climate, 4, 2014, p. A19.

7. G. Drukarev, "On the Mean Energy of Electrons Released in the Ionization of Gas, 1," Journal of Physics (USSR), 10, 1946, p. 81.

8. G. V. Khazanov, Kinetic Theory of the Inner Magnetospheric Plasma, Vol. 372, New York, Springer Science \& Business Media, 2010.

9. R. Schunk and N. Andrew, Ionospheres: Physics, Plasma Physics, and Chemistry, Cambridge, UK, Cambridge University Press, 2009.

10. D. Bilitza, "International Reference Ionosphere 2000," Radio Science, 36, 2, 2001, pp. 261-275.

11. J. T. D. Sartori, "Expanding the Reach of Ionosphere Real-Time Measurements: Proposal of a Virtual Ionosonde Concept," XXXIV URSI General Assembly and Scientific Symposium (URSI GASS 2021), XXXX, August 30, 2021, pp. XXX-XXX.

12. J. Coles and N. Eales, "The History of Comparative Anatomy: A Statistical Analysis of Scientific Literature," Science Progress, 11, 1917, pp. 578-596.

13. L. Björneborn, Small-World Link Structures Across an Academic Web Space: A Library and Information Science Approach, Aalborg, Denmark, Royal School of Library and Information Science, 2004.

14. L. Egghe, "Expansion of the Field of Informetrics: Origins and Consequences." Information Processing and Management, 41, 6, 2005, pp. 1311-1316.

15. W. Glanzel, "Bibliometrics as a Research Field: A Course on Theory and Application of Bibliometric Indicators," 2003.

16. J. E. Hirsch, "An Index to Quantify an Individual's Scientific Research Output," Proceedings of the National Academy of Sciences, 102, 46, 2005, pp. 16569-16572.

17. O. Azeroual, S. Gunter, and W. Jürgen, "Data Measurement in Research Information Systems: Metrics for the Evaluation of Data Quality," Scientometrics, 115, 3, 2018, pp. 1271-1290.

18. V. T. Verhulst, D. Altadill, J. Mielich, B. Reinischd, I. Galkin, A. et al., "Vertical and Oblique HF Sounding With a Network of Synchronised Ionosondes," Advances in Space Research, 60, 8, 2017, pp. 1644-1656. 